\title{
A Problem with Parameter for the Integro-Differential Equations
}

\author{
Elmira A. Bakirova ${ }^{a, b}$, Anar T. Assanova ${ }^{a, b}$ and \\ Zhazira M. Kadirbayeva ${ }^{a, b, c}$ \\ ${ }^{a}$ Institute of Mathematics and Mathematical Modeling \\ Pushkin Str. 125, Almaty, Kazakhstan \\ ${ }^{b}$ Institute of Information and Computational Technologies \\ Pushkin Str. 125, Almaty, Kazakhstan \\ ${ }^{c}$ International Information Technology University \\ Jandossov Str. 34A, Almaty, Kazakhstan \\ E-mail(corresp.): anartasan@gmail.com; assanova@math.kz \\ E-mail: bakirova1974@mail.ru \\ E-mail: apelman86pm@mail.ru
}

Received January 22, 2020; revised October 7, 2020; accepted October 7, 2020

\begin{abstract}
The article proposes a numerically approximate method for solving a boundary value problem for an integro-differential equation with a parameter and considers its convergence, stability, and accuracy. The integro-differential equation with a parameter is approximated by a loaded differential equation with a parameter. A new general solution to the loaded differential equation with a parameter is introduced and its properties are described. The solvability of the boundary value problem for the loaded differential equation with a parameter is reduced to the solvability of a system of linear algebraic equations with respect to arbitrary vectors of the introduced general solution. The coefficients and the right-hand sides of the system are compiled through solutions of the Cauchy problems for ordinary differential equations. Algorithms are proposed for solving the boundary value problem for the loaded differential equation with a parameter. The relationship between the qualitative properties of the initial and approximate problems is established, and estimates of the differences between their solutions are given.
\end{abstract}

Keywords: integro-differential equation, problem with parameter, $\delta_{m}(\theta)$ general solution, solvability criteria, algorithm, numerical solution.

AMS Subject Classification: 34K06; 34K35; 45J05; 45J99.

Copyright (c) 2021 The Author(s). Published by Vilnius Gediminas Technical University This is an Open Access article distributed under the terms of the Creative Commons Attribution License (http://creativecommons.org/licenses/by/4.0/), which permits unrestricted use, distribution, and reproduction in any medium, provided the original author and source are credited. 


\section{Introduction}

The theory of control problems for a system of ordinary differential equations and for a system of integro-differential equations in partial derivatives, with parameters, is rapidly developing and used in various fields of applied mathematics, biophysics, biomedicine, chemistry, etc. Control problems, also called as boundary value problems with parameters and parameter identification problems for systems of ordinary differential and integro-differential equations with parameters, are intensively studied by many authors $[3,4,8,9,17,18,19,20,24$, 25]. To find solutions to these problems, methods of the qualitative theory of differential equations, variational calculus and optimization theory, the method of upper and lower solutions, etc. were used. Note that the problems of determining effective criteria for unique solvability and constructing numerical algorithms for finding optimal solutions to control problems for systems of ordinary differential and integro-differential equations with parameters are still relevant.

In this article, we extend the methods and results of $[14,15]$ to a boundary value problem for integro-differential equations with parameters. The solvability conditions for boundary value problems for a system of integro-differential equations with parameters are established. A numerically approximate method for solving the investigated boundary value problem is constructed, and its convergence, stability, and accuracy are investigated.

Consider the boundary value problem for the integro-differential equation with a parameter

$$
\begin{aligned}
& \frac{d x}{d t}=A(t) x+\int_{0}^{T} K(t, \tau) x(\tau) d \tau+A_{0}(t) \mu+f(t), x \in R^{n}, \mu \in R^{l}, t \in(0, T), \\
& B_{0} \mu+B x(0)+C x(T)=d, \quad d \in R^{n+l} .
\end{aligned}
$$

Here the $(n \times n)$ matrices $A(t)$ and $K(t, \tau)$ are continuous on $[0, T]$ and $[0, T] \times$ $[0, T]$, respectively; the $(n \times l)$ matrix $A_{0}(t)$ is continuous on $[0, T]$, the $n$ vector $f(t)$ is continuous on $[0, T]$.

Let $C\left([0, T], R^{n}\right)$ be the space of continuous functions $x:[0, T] \rightarrow R^{n}$ with the norm $\|x\|_{1}=\max _{t \in[0, T]}\|x(t)\|=\max _{i=\overline{1, n}} \max _{t \in[0, T]}\left|x_{i}(t)\right|$.

Solution to problem with parameter in Equations (1.1)-(1.2) is a pair $\left(x^{*}(t), \mu^{*}\right)$, where the function $x^{*}(t) \in C\left([0, T], R^{n}\right)$ is continuously differentiable on $(0, T)$, the parameter $\mu^{*} \in R^{l}$, satisfies integro-differential equation (1.1) and boundary condition (1.2). Based on the parameterization method [16], the solvability and unique solvability criteria for problem (1.1)-(1.2) without a parameter are set in $[11,12,13,16]$. Algorithms for the parameterization method for solving this problem are proposed in $[14,15]$.

The aim of this paper is to construct approximate and numerical methods for solving the problem with parameter in (1.1)-(1.2) and to ensure their convergence, stability, and accuracy.

Section 2 is devoted to a loaded differential equation with a parameter. By the Simpson formula, the integral term of Equation (1.1) is replaced by the integral sum, and problem (1.1)-(1.2) reduces to the problem for a loaded 
differential equation with parameter. A new approach to the general solution of a loaded differential equation with a parameter is proposed. A new $\delta_{m}(\theta)$ general solution exists for all loaded linear nonhomogeneous differential equations with parameter and depends on the $m+1$ arbitrary vectors $\lambda_{r} \in R^{n}$. We study the properties of $\delta_{m}(\theta)$ general solution and obtain solvability criteria for loaded differential equations with a parameter. The condition for the existence of a classical general solution is also established. Numerical and approximate methods for compilation of the $\delta_{m}(\theta)$ general solution are proposed.

The employment of the $\delta_{m}(\theta)$ general solution for solving the problem with a parameter for a loaded differential equation is given in Section 3. Substituting the expressions of the $\delta_{m}(\theta)$ general solution into the boundary condition (1.2) and the continuity conditions for solutions at loaded points, we obtain a system of linear algebraic equations with respect to $\lambda_{r} \in R^{n}, r=\overline{1, m+1}$. Invertibility of $Q_{*}\left(\delta_{m}(\theta)\right)$, the matrix of the compiled system, is equivalent to the wellposedness of the problem with a parameter for the loaded differential equation. The coefficients and the right-hand sides of this system are constructed through solutions to the Cauchy problems for ordinary differential equations on the subintervals $\left[\theta_{r-1}, \theta_{r}\right], r=\overline{1, m}$. Algorithms for solving the problem with a parameter for the loaded differential equation are proposed.

The interrelation between the unique solvability of the initial boundary value problem and the unique solvability of the approximate boundary value problem is established in Section 4. Estimates for the differences between their solutions are given.

Section 5 presents methods for solving the problem with parameter (1.1)(1.2). The interval $[0, T]$ is divided into $2 N$ parts with a step $h>0: 2 N h=T$. Equation (1.1) is replaced by a loaded differential equation, and problem (1.1)(1.2) is approximated by a problem with a parameter for a loaded differential equation.

The results of Section 4 and the continuity of the kernel $K(t, \tau)$ on $[0, T] \times$ $[0, T]$ give uniform convergence of approximate solutions of the problem to the solution of problem $(1.1)-(1.2)$ on $[0, T]$ as $h \rightarrow 0$. It is shown that the convergence is of the fourth order with respect to $h$ if data of problem (1.1)(1.2) are sufficiently smooth.

For an approximate problem with a parameter, a system of linear algebraic equations with respect to arbitrary vectors of a new general solution is composed. It is shown that the conditionality number of this system increases linearly relative to $2 N$ if the approximate problem with the parameter is wellposed. This property of the system guarantees its stable solution. Cauchy problems for ordinary differential equations on subintervals are the main auxiliary problems in the proposed methods. If we choose an approximate method for solving these problems, we obtain an approximate method for solving the problem with parameter (1.1)-(1.2).

Numerical methods for solving Cauchy problems give numerical methods for solving the problem with parameter (1.1)-(1.2). 


\section{Approximate loaded linear differential equation with parameter: new general solution and its properties}

Replacing the integral term of Equation (1.1) by a suitable integral sum, we obtain the loaded differential equation with parameter

$$
\frac{d x}{d t}=A(t) x+\sum_{j=1}^{m+1} K_{j}(t) x\left(\theta_{j-1}\right)+A_{0}(t) \mu+f(t),
$$

where $t \in(0, T), x \in R^{n}, \mu \in R^{l}$, the $(n \times n)$ matrices $K_{j}(t), j=\overline{1, m+1}$, are continuous on $[0, T]$ and $0=\theta_{0}<\theta_{1}<\ldots<\theta_{m-1}<\theta_{m}=T$.

Solution to Equation (2.1) is a pair $\left(x^{*}(t), \mu^{*}\right)$, where the function $x^{*}(t) \in$ $C\left([0, T], R^{n}\right)$ is continuously differentiable on $(0, T)$, the parameter $\mu^{*} \in R^{l}$ satisfies the equation for all $t \in(0, T)$.

Equation (2.1) is also referred to as a differential boundary equation. Loaded differential equations frequently are used in the applied mathematics. Particularly, in [21]- [22], they are used to describe the problems of long-term forecasting and control of groundwater level in the soil moisture. Various problems for these equations and methods for solving them are studied in $[1,2,5,6,18,23]$. The Fredholm integro-differential equation with a parameter has several features that should be considered when methods for research and solving problems with Equation (1.1) are created. The existence of an unsolvable Fredholm integro-differential equation with a parameter is one of such features. Another feature is that the Cauchy problem for Equation (1.1) can be unsolvable, although the problem with parameters for this equation has a unique solution. These features are also the characteristics of loaded differential equations with a parameter.

Suppose $(x(t, c), \mu(c))$ is a solution to Equation (2.1) for all $c \in R^{n}$, and each solution $(\widetilde{x}(t), \mu(c))$ of Equation (2.1) coincides with the function $(x(t, c), \mu(c))$ for a unique $c=\widetilde{c}$. Then the pair $(x(t, c), \mu(c))$ is said to be the classical general solution of the loaded linear differential equation with parameter $(2.1)$ if it exists for all $f(t) \in C\left([0, T], R^{n}\right)$. General solution is one of the main tools for research and solving problems for differential and integro-differential equations. Since there are unsolvable loaded linear differential equations, the classical general solution also exists not for all equations (2.1). Therefore, we propose a new concept for the general solution of a loaded differential equation with a parameter.

Let $C\left([0, T], \theta, R^{n m}\right)$ denote the space of function systems $x[t]=\left(x_{1}(t)\right.$, $\left.x_{2}(t), \ldots, x_{m}(t)\right)$, where $x_{r}:\left[\theta_{r-1}, \theta_{r}\right) \rightarrow R^{n}$ are continuous and have finite left-side limits $\lim _{t \rightarrow \theta_{r}-0} x_{r}(t)$ for all $r=\overline{1, m}$, with norm

$$
\|x[\cdot]\|_{2}=\max _{r=\overline{1, m}} \sup _{t \in\left[\theta_{r-1}, \theta_{r}\right)}\left\|x_{r}(t)\right\| .
$$

Let $(x(t), \mu)$ be the solution of the loaded differential equation with parameter $(2.1)$, and $x_{r}(t)$ be the restriction of the function $x(t)$ to the sub-interval $\left[\theta_{r-1}, \theta_{r}\right)$, i.e. $x_{r}(t)=x(t), t \in\left[\theta_{r-1}, \theta_{r}\right), r=\overline{1, m}$. Then the pair $(x[t], \mu)$, where the function system $x[t]=\left(x_{1}(t), x_{2}(t), \ldots, x_{m}(t)\right)$ belongs to 
$C\left([0, T], \theta, R^{n m}\right), \mu \in R^{l}$ and satisfies the system of loaded differential equations with parameter:

$$
\frac{d x_{r}}{d t}=A(t) x_{r}+\sum_{j=1}^{m} K_{j}(t) x_{j}\left(\theta_{j-1}\right)+K_{m+1}(t) x\left(\theta_{m}\right)+A_{0}(t) \mu+f(t),
$$

where $t \in\left(\theta_{r-1}, \theta_{r}\right), r=\overline{1, m}, x\left(\theta_{m}\right)$ is the value of function $x(t)$ at the endpoint of interval: $t=\theta_{m}=T$.

A triple $\left(\widetilde{x}[t], \widetilde{x}\left(\theta_{m}\right), \mu\right)$ with $\widetilde{x}[t]=\left(\widetilde{x}_{1}(t), \widetilde{x}_{2}(t), \ldots, \widetilde{x}_{m}(t)\right) \in C\left([0, T], \theta, R^{n m}\right)$, and $\widetilde{x}\left(\theta_{m}\right) \in R^{n}$, and $\mu \in R^{l}$ that satisfies Equation (2.2) is called a solution to Equation (2.2).

We introduce the parameters $\lambda_{r}=x_{r}\left(\theta_{r-1}\right), r=\overline{1, m}$, and $\lambda_{m+1}=x\left(\theta_{m}\right)$, and $\lambda_{m+2}=\mu$. We also introduce an unknown functions $u_{r}:\left[\theta_{r-1}, \theta_{r}\right) \rightarrow R^{n}$, $r=\overline{1, m}$. By substituting $u_{r}(t)=x_{r}(t)-\lambda_{r}$ at every $r$ th interval $\left[\theta_{r-1}, \theta_{r}\right)$, we obtain a system of ordinary differential equations with parameters

$$
\frac{d u_{r}}{d t}=A(t)\left(u_{r}+\lambda_{r}\right)+\sum_{j=1}^{m+1} K_{j}(t) \lambda_{j}+A_{0}(t) \lambda_{m+2}+f(t), \quad t \in\left(\theta_{r-1}, \theta_{r}\right),
$$

and the initial conditions

$$
u_{r}\left(\theta_{r-1}\right)=0, \quad r=\overline{1, m} .
$$

For any fixed $\hat{\lambda}=\left(\hat{\lambda}_{1}, \hat{\lambda}_{2}, \ldots, \hat{\lambda}_{m+1}, \hat{\lambda}_{m+2}\right) \in R^{n(m+1)+l}$ and $r=\overline{1, m}$, the Cauchy problem $(2.3)-(2.4)$ has a unique solution $u_{r}(t, \hat{\lambda})$ on the subinterval $\left[\theta_{r-1}, \theta_{r}\right)$, and the function system $u[t, \hat{\lambda}]=\left(u_{1}(t, \hat{\lambda}), u_{2}(t, \hat{\lambda}), \ldots, u_{m}(t, \hat{\lambda})\right)$ belongs to $C\left([0, T], \theta, R^{n m}\right)$. The function system $u[t, \hat{\lambda}]$ is called the solution to the Cauchy problem (2.3)-(2.4) with $\lambda=\hat{\lambda}$. The system of loaded differential equations with parameter (2.2) is equivalent to Cauchy problem with parameters $(2.3)-(2.4)$ in the following sense.

Let the triple $\left(\widetilde{x}[t]=\left(\widetilde{x}_{1}(t), \widetilde{x}_{2}(t), \ldots, \widetilde{x}_{m}(t)\right), \widetilde{x}\left(\theta_{m}\right), \widetilde{\mu}\right)$ be a solution of Equation (2.2). Choose the parameters $\widetilde{\lambda}_{r}=\widetilde{x}_{r}\left(\theta_{r-1}\right), \quad r=\overline{1, m}$, and $\widetilde{\lambda}_{m+1}=$ $\widetilde{x}\left(\theta_{m}\right)$, and $\widetilde{\lambda}_{m+2}=\widetilde{\mu}$. Then the function system $u[t, \widetilde{\lambda}]=\left(u_{1}(t, \widetilde{\lambda}), u_{2}(t, \widetilde{\lambda}), \ldots\right.$, $\left.u_{m}(t, \widetilde{\lambda})\right)$, where $u_{r}(t, \widetilde{\lambda})=\widetilde{x}_{r}(t)-\widetilde{\lambda}_{r}, r=\overline{1, m}$, is a solution to the Cauchy problem (2.3)-(2.4) with $\lambda=\widetilde{\lambda}=\left(\widetilde{\lambda}_{1}, \widetilde{\lambda}_{2}, \ldots, \widetilde{\lambda}_{m+1}, \widetilde{\lambda}_{m+2}\right) \in R^{n(m+1)+l}$. And vice versa, if the function system $u\left[t, \lambda^{*}\right]=\left(u_{1}\left(t, \lambda^{*}\right), u_{2}\left(t, \lambda^{*}\right), \ldots, u_{m}\left(t, \lambda^{*}\right)\right) \in$ $C\left([0, T], \theta, R^{n m}\right)$ is a solution to the Cauchy problem $(2.3)-(2.4)$ with $\lambda=\lambda^{*}=$ $\left(\lambda_{1}^{*}, \lambda_{2}^{*}, \ldots, \lambda_{m+1}^{*}, \lambda_{m+2}^{*}\right) \in R^{n(m+1)+l}$, then the triple $\left(x^{*}[t]=\left(x_{1}^{*}(t), x_{2}^{*}(t), \ldots\right.\right.$, $\left.\left.x_{m}^{*}(t)\right), x^{*}\left(\theta_{m}\right), \mu^{*}\right)$ with $x_{r}^{*}(t)=\lambda_{r}^{*}+u_{r}\left(t, \lambda^{*}\right), r=\overline{1, m}$, and $x^{*}\left(\theta_{m}\right)=\lambda_{m+1}^{*}$, and $\mu^{*}=\lambda_{m+2}^{*}$ is a solution of Equation (2.2).

Next, a new concept of a general solution of a loaded differential equation with a parameter is introduced.

Definition 1 . Let $u[t, \lambda]=\left(u_{1}(t, \lambda), u_{2}(t, \lambda), \ldots, u_{m}(t, \lambda)\right)$ be a solution to the Cauchy problem $(2.3)-(2.4)$ with $\lambda=\left(\lambda_{1}, \lambda_{2}, \ldots, \lambda_{m+1}, \lambda_{m+2}\right) \in R^{n(m+1)+l}$. Then the pair $\left(x\left(\delta_{m}(\theta), \lambda, t\right), \mu\left(\delta_{m}(\theta), \lambda\right)\right)$, where the function $x\left(\delta_{m}(\theta), \lambda, t\right)$ and the parameter $\mu\left(\delta_{m}(\theta), \lambda\right)$ are given by the equalities

$$
x\left(\delta_{m}(\theta), \lambda, t\right)=\lambda_{r}+u_{r}(t, \lambda) \text { for } t \in\left[\theta_{r-1}, \theta_{r}\right), \quad r=\overline{1, m},
$$


and $x\left(\delta_{m}(\theta), \lambda, T\right)=\lambda_{m+1}, \mu\left(\delta_{m}(\theta), \lambda\right)=\lambda_{m+2}$ is called a $\delta_{m}(\theta)$ general solution for a loaded differential equation with parameter (2.1).

As follows from Definition 1, the $\delta_{m}(\theta)$ general solution depends on the $m+2$ arbitrary vectors $\lambda_{r} \in R^{n}, r=\overline{1, m+1}, \lambda_{m+2} \in R^{l}$, and satisfies the loaded differential equation with parameter (2.1) for all $t \in(0, T) \backslash\left\{\theta_{p}, p=\overline{1, m-1}\right\}$.

Let $X_{r}(t)$ be the fundamental matrix of the ordinary differential equation

$$
\frac{d x}{d t}=A(t) x, \quad t \in\left[\theta_{r-1}, \theta_{r}\right], \quad r=\overline{1, m} .
$$

Then the unique solution to the Cauchy problem (2.3)-(2.4) can be written as

$$
\begin{aligned}
& u_{r}(t, \lambda)=X_{r}(t) \int_{\theta_{r-1}}^{t} X_{r}^{-1}(\tau)\left(A(\tau)+K_{r}(\tau)\right) d \tau \lambda_{r} \\
& +\sum_{\substack{j=1 \\
j \neq r}}^{m+1} X_{r}(t) \int_{\theta_{r-1}}^{t} X_{r}^{-1}(\tau) K_{j}(\tau) d \tau \lambda_{j}+X_{r}(t) \int_{\theta_{r-1}}^{t} X_{r}^{-1}(\tau) A_{0}(\tau) d \tau \lambda_{m+2} \\
& +X_{r}(t) \int_{\theta_{r-1}}^{t} X_{r}^{-1}(\tau) f(\tau) d \tau, \quad t \in\left[\theta_{r-1}, \theta_{r}\right), \quad r=\overline{1, m}
\end{aligned}
$$

Therefore, from (2.5) we obtain the following representation of the $\delta_{m}(\theta)$ general solution:

$$
\begin{aligned}
& x\left(\delta_{m}(\theta), \lambda, t\right)=\sum_{j=1}^{m+1} \alpha_{r j}\left(\delta_{m}(\theta), t\right) \lambda_{j}+\alpha_{r}^{0}\left(\delta_{m}(\theta), t\right) \lambda_{m+2}+\beta_{r}\left(\delta_{m}(\theta), t\right) \\
& \quad t \in\left[\theta_{r-1}, \theta_{r}\right), \quad r=\overline{1, m}, \\
& x\left(\delta_{m}(\theta), \lambda, T\right)=\lambda_{m+1} \\
& \mu\left(\delta_{m}(\theta), \lambda\right)=\lambda_{m+2}
\end{aligned}
$$

with

$$
\begin{aligned}
& \alpha_{r r}\left(\delta_{m}(\theta), t\right)=I+X_{r}(t) \int_{\theta_{r-1}}^{t} X_{r}^{-1}(\tau)\left(A(\tau)+K_{r}(\tau)\right) d \tau \\
& t \in\left[\theta_{r-1}, \theta_{r}\right), r=\overline{1, m} \\
& \alpha_{r j}\left(\delta_{m}(\theta), t\right)=X_{r}(t) \int_{\theta_{r-1}}^{t} X_{r}^{-1}(\tau) K_{j}(\tau) d \tau, \\
& t \in\left[\theta_{r-1}, \theta_{r}\right), j=\overline{1, m+1}, j \neq r, \\
& \alpha_{r}^{0}\left(\delta_{m}(\theta), t\right)=X_{r}(t) \int_{\theta_{r-1}}^{t} X_{r}^{-1}(\tau) A_{0}(\tau) d \tau, \quad t \in\left[\theta_{r-1}, \theta_{r}\right), \quad r=\overline{1, m}, \\
& \beta_{r}\left(\delta_{m}(\theta), t\right)=X_{r}(t) \int_{\theta_{r-1}}^{t} X_{r}^{-1}(\tau) f(\tau) d \tau, \quad t \in\left[\theta_{r-1}, \theta_{r}\right), \quad r=\overline{1, m},
\end{aligned}
$$

where $I$ is the identity matrix of dimension $n$.

The $(n \times n)$ matrices $\alpha_{r j}\left(\delta_{m}(\theta), t\right), \quad r=\overline{1, m}, \quad j=\overline{1, m+1}$, the $(n \times l)$ matrices $\alpha_{r}^{0}\left(\delta_{m}(\theta), t\right), \quad r=\overline{1, m}$, and $n$ vectors $\beta_{r}\left(\delta_{m}(\theta), t\right), \quad r=\overline{1, m}$, are called the coefficients and right sides of $\delta_{m}(\theta)$ general solution, respectively. 
Theorem 1. Let a pair $(\widetilde{x}(t), \widetilde{\mu})$, where the function $\widetilde{x}(t)$ is piecewise continuous on $[0, T]$ with possible discontinuity points $t=\theta_{r}, r=\overline{1, m}$ and the parameter $\widetilde{\mu} \in R^{l}$, be given, and the pair $\left(x\left(\delta_{m}(\theta), \lambda, t\right), \mu\left(\delta_{m}(\theta), \lambda\right)\right)$ be the $\delta_{m}(\theta)$ general solution to Equation (2.1). Suppose that the function $\widetilde{x}(t)$ has a continuous derivative and satisfies Equation (2.1) for all $t \in(0, T) \backslash\left\{\theta_{p}, p=\overline{1, m-1}\right\}$. Then there is a unique $\widetilde{\lambda}=\left(\widetilde{\lambda}_{1}, \widetilde{\lambda}_{2} \ldots, \widetilde{\lambda}_{m+1}, \widetilde{\lambda}_{m+2}\right) \in R^{n(m+1)+l}$ such that the equality $x\left(\delta_{m}(\theta), \widetilde{\lambda}, t\right)=\widetilde{x}(t)$ holds for all $t \in[0, T]$ and $\mu\left(\delta_{m}(\theta), \lambda\right)=\widetilde{\mu}$.

Proof. Let a pair $(\widetilde{x}(t), \widetilde{\mu})$, where the function $\widetilde{x}(t)$ is piecewise continuous on $[0, T]$, the parameter $\widetilde{\mu} \in R^{l}$, be given, and $\widetilde{x}[t]=\left(\widetilde{x}_{1}(t), \widetilde{x}_{2}(t), \ldots, \widetilde{x}_{m}(t)\right)$ be a function system of restrictions of the function $\widetilde{x}(t)$ to $\left[\theta_{r-1}, \theta_{r}\right), r=\overline{1, m}$. Under the conditions of the Theorem, the triple $\left(\widetilde{x}[t], \widetilde{x}\left(\theta_{m}\right), \widetilde{\mu}\right)$ is a solution to Equations (2.2). Assign the parameter $\widetilde{\lambda}=\left(\widetilde{\lambda}_{1}, \widetilde{\lambda}_{2} \ldots, \widetilde{\lambda}_{m+1}, \widetilde{\lambda}_{m+2}\right) \in$ $R^{n(m+1)+l}$ with $\widetilde{\lambda}_{r}=\widetilde{x}_{r}\left(\theta_{r-1}\right), r=\overline{1, m}$, and $\widetilde{\lambda}_{m+1}=\widetilde{x}\left(\theta_{m}\right)$, and $\widetilde{\lambda}_{m+2}=\widetilde{\mu}$, for the pair $(\widetilde{x}(t), \widetilde{\mu})$.

We solve Cauchy problem (2.3)-(2.4) with $\lambda=\widetilde{\lambda}$ and find $u[t, \widetilde{\lambda}]=\left(u_{1}(t, \tilde{\lambda})\right.$, $\left.u_{2}(t, \tilde{\lambda}), \ldots, u_{m}(t, \tilde{\lambda})\right)$. Then, due to the relations between the solution of Equation (2.2) and the solution of the Cauchy problem (2.3)-(2.4), we obtain:

$$
\begin{aligned}
\widetilde{x}(t) & =\widetilde{x}_{r}(t)=\widetilde{\lambda}_{r}+u_{r}(t, \widetilde{\lambda})=x\left(\delta_{m}(\theta), \widetilde{\lambda}, t\right), t \in\left[\theta_{r-1}, \theta_{r}\right), r=\overline{1, m}, \\
\widetilde{x}(T) & =\widetilde{x}\left(\theta_{m}\right)=\widetilde{\lambda}_{m+1}=x\left(\delta_{m}(\theta), \widetilde{\lambda}, T\right), \quad \widetilde{\mu}=\widetilde{\lambda}_{m+2}=\mu\left(\delta_{m}(\theta), \widetilde{\lambda}\right) .
\end{aligned}
$$

To show that $\tilde{\lambda}$ is unique, suppose that $\lambda^{*}=\left(\lambda_{1}^{*}, \ldots, \lambda_{m+1}^{*}, \lambda_{m+2}^{*}\right) \in R^{n(m+1)+l}$ is another parameter such that $\widetilde{x}(t)=x\left(\delta_{m}(\theta), \lambda^{*}, t\right)$ for all $t \in[0, T]$, and $\widetilde{\mu}=\mu\left(\delta_{m}(\theta), \lambda^{*}\right)$.

Then, according to Definition 1 , we have $\widetilde{x}(t)=\widetilde{x}_{r}(t)=\lambda_{r}^{*}+u_{r}\left(t, \lambda^{*}\right)$ for $t \in\left[\theta_{r-1}, \theta_{r}\right), r=\overline{1, m}, \widetilde{x}(T)=\lambda_{m+1}^{*}, \widetilde{\mu}=\lambda_{m+2}^{*}$, where the function system $u\left[t, \lambda^{*}\right]=\left(u_{1}\left(t, \lambda^{*}\right), u_{2}\left(t, \lambda^{*}\right), \ldots, u_{m}\left(t, \lambda^{*}\right)\right)$ is a solution to the Cauchy problem (2.3)-(2.4) with $\lambda=\lambda^{*} \in R^{n(m+1)+l}$. Now, using the initial conditions (2.4) and the way of choosing $\widetilde{\lambda} \in R^{n(m+1)+l}$, we get $\widetilde{\lambda}_{r}=\widetilde{x}_{r}\left(\theta_{r-1}\right)=\lambda_{r}^{*}+$ $u_{r}\left(\theta_{r-1}, \lambda^{*}\right)=\lambda_{r}^{*}, r=\overline{1, m}$, and $\widetilde{\lambda}_{m+1}=\widetilde{x}\left(\theta_{m}\right)=\widetilde{x}(T)=\lambda_{m+1}^{*}$, and $\widetilde{\lambda}_{m+2}=$ $\widetilde{\mu}=\lambda_{m+2}^{*}$. Theorem 1 is proved.

Corollary 1. Let a pair $\left(x^{*}(t), \mu^{*}\right)$ be a solution to Equation (2.1), and a pair $\left(x\left(\delta_{m}(\theta), \lambda, t\right), \mu\left(\delta_{m}(\theta), \lambda\right)\right)$ be a $\delta_{m}(\theta)$ general solution to Equation (2.1). Then there exists a unique $\lambda^{*}=\left(\lambda_{1}^{*}, \lambda_{2}^{*}, \ldots, \lambda_{m+1}^{*}, \lambda_{m+2}^{*}\right) \in R^{n(m+1)+l}$ such that the equality $x\left(\delta_{m}(\theta), \lambda^{*}, t\right)=x^{*}(t)$ holds for all $t \in[0, T]$ and $\mu\left(\delta_{m}(\theta), \lambda\right)=\mu^{*}$.

If a pair $(x(t), \mu)$ is a solution of Equation $(2.1)$, and $x[t]=\left(x_{1}(t), x_{2}(t), \ldots\right.$, $\left.x_{m}(t)\right)$ is a function system consisting of restrictions of the function $x(t)$ to the sub-intervals $\left[\theta_{r-1}, \theta_{r}\right), \quad r=\overline{1, m}$, then the following equations hold:

$$
\begin{aligned}
\lim _{t \rightarrow \theta_{p}-0} x_{p}(t) & =x_{p+1}\left(\theta_{p}\right), \quad p=\overline{1, m-1}, \\
\lim _{t \rightarrow T-0} x_{m}(t) & =x\left(\theta_{m}\right) .
\end{aligned}
$$

These equations are called continuity conditions for solution of Equation (2.1). 
Theorem 2. Let the triple $\left(x[t], x\left(\theta_{m}\right), \mu\right)$ with $x[t]=\left(x_{1}(t), x_{2}(t), \ldots, x_{m}(t)\right)$ $\in C\left([0, T], \theta, R^{n m}\right)$ and $x\left(\theta_{m}\right) \in R^{n}$, and $\mu \in R^{l}$ satisfy Equation (2.2) and continuity conditions (2.13)-(2.14). Then the pair $\left(x^{*}(t), \mu^{*}\right)$, where the function $x^{*}(t)$, given by the equalities $x^{*}(t)=x_{r}(t)$ for $t \in\left[\theta_{r-1}, \theta_{r}\right), \quad r=\overline{1, m}$, and $x^{*}(T)=x\left(\theta_{m}\right)$, and $\mu^{*}=\mu$, is a solution of the loaded differential equation with parameter $(2.1)$.

Proof. Equations (2.13) and (2.14) yield the continuity of the function $x^{*}(t)$ on $[0, T]$. Since the triple $\left(x[t], x\left(\theta_{m}\right), \mu\right)$ satisfies Equation (2.4), the pair $\left(x^{*}(t), \mu^{*}\right)$, where the function $x^{*}(t)$ has a continuous derivative and satisfies Equation (2.1) for all $t \in(0, T) \backslash\left\{\theta_{p}, p=\overline{1, m-1}\right\}$, and $\mu=\mu^{*}$. The existence and continuity of $\dot{x}^{*}(t)$ at the points $t=\theta_{p}, p=\overline{1, m-1}$, follow from the following relations:

$$
\begin{aligned}
\lim _{t \rightarrow \theta_{p}-0} \dot{x}^{*}(t) & =A\left(\theta_{p}\right) x^{*}\left(\theta_{p}\right)+\sum_{j=1}^{m+1} K_{j}\left(\theta_{p}\right) x^{*}\left(\theta_{j-1}\right)+A_{0}\left(\theta_{p}\right) \mu^{*}+f\left(\theta_{p}\right) \\
& =\lim _{t \rightarrow \theta_{p}+0} \dot{x}^{*}(t), \quad p=\overline{1, m-1}
\end{aligned}
$$

Relations (2.15) mean that the pair $\left(x^{*}(t), \mu^{*}\right)$ satisfies the loaded differential equation with parameter on the interior loaded points of $[0, T]$. Theorem 2 is proved.

It is clear that the boundary value problem might be considered only for solvable loaded differential equations with a parameter. Thus, we apply the $\delta_{m}(\theta)$ general solution to establish the solvability criteria for Equation (2.1). Substituting the appropriate expressions from (2.6) and (2.7) into the continuity conditions (2.13) and (2.14), we obtain the system of $m n$ linear algebraic equations with respect to the $m+2$ unknown vectors $\lambda_{j} \in R^{n}, j=\overline{1, m+1}$, $\lambda_{m+2} \in R^{l}$

$$
\begin{gathered}
\sum_{\substack{j=1 \\
j \neq r+1}}^{m+1} \alpha_{r j}\left(\delta_{m}(\theta), \theta_{r}\right) \lambda_{j}- \\
=-\beta_{r}\left(\delta_{m}(\theta), \theta_{r}\right), \quad r=\overline{1, m}
\end{gathered}
$$

Let $D_{*}\left(\delta_{m}(\theta)\right)$ denote the $n m \times(n(m+1)+l)$ matrix corresponding to the left-hand side of (2.16). We rewrite (2.16) in the form:

$$
D_{*}\left(\delta_{m}(\theta)\right) \lambda=-\beta_{*}\left(\delta_{m}(\theta), f\right),
$$

where $\beta_{*}\left(\delta_{m}(\theta), f\right)=\left(\beta_{1}\left(\delta_{m}(\theta), \theta_{1}\right), \beta_{2}\left(\delta_{m}(\theta), \theta_{2}\right), \ldots, \beta_{m}\left(\delta_{m}(\theta), \theta_{m}\right)\right) \in R^{n m}$.

Theorems 1, 2 and well-known statements of linear algebra lead to the following assertion.

Theorem 3. A loaded linear differential equation with parameter (2.1) is solvable if and only if the vector $\beta_{*}\left(\delta_{m}(\theta), f\right)$ is orthogonal to the kernel of the transposed matrix $\left(D_{*}\left(\delta_{m}(\theta)\right)\right)^{\prime}$, i.e. iff the equality

$$
\left(\beta_{*}\left(\delta_{m}(\theta), f\right), \eta\right)=0
$$


is valid for $\forall \eta \in \operatorname{Ker}\left(D_{*}\left(\delta_{m}(\theta)\right)\right)^{\prime}$, where $(\cdot, \cdot)$ is the inner product in $R^{n m}$.

Equation (2.17) and properties of the general solution allow us to obtain the following statement on the existence of a classical general solution to Equation (2.1).

Theorem 4. A loaded linear differential equation with parameter (2.1) has a classical general solution if $\operatorname{rank} D_{*}\left(\delta_{m}(\theta)\right)=m n$.

Proof. Consider the system of $m n$ linear algebraic equations (2.17). By assumption, the matrix $D_{*}\left(\delta_{m}(\theta)\right)$ has $m n$ linearly independent columns. We denote by $D_{0}^{*}\left(\delta_{m}(\theta)\right)$ the $m n \times m n$ matrix consisting of these columns, and by $D_{*}^{*}\left(\delta_{m}(\theta)\right)$ denote the $m n \times(n+l)$ matrix consisting of the remaining columns. We rewrite system $(2.17)$ in the form:

$$
D_{0}^{*}\left(\delta_{m}(\theta)\right) \lambda_{0}+D_{*}^{*}\left(\delta_{m}(\theta)\right) \lambda^{*}=-\beta_{*}\left(\delta_{m}(\theta), f\right),
$$

where $\lambda_{0} \in R^{n m}$ and $\lambda^{*} \in R^{n+l}$ are the vectors composed of the coordinates of the vector $\lambda \in R^{n(m+1)+l}$ corresponding to our decomposition of matrix $D_{*}\left(\delta_{m}(\theta)\right)$. Since the matrix $D_{0}^{*}\left(\delta_{m}(\theta)\right): R^{n m} \rightarrow R^{n m}$ is invertible, the vector $\lambda_{0} \in R^{n m}$ is uniquely determined by $(2.18)$ :

$$
\lambda_{0}=\left(D_{0}^{*}\left(\delta_{m}(\theta)\right)\right)^{-1}\left(-\beta_{*}\left(\delta_{m}(\theta), f\right)-D_{*}^{*}\left(\delta_{m}(\theta)\right) \lambda^{*}\right) .
$$

Let us take $\lambda^{*}$ as an arbitrary vector $c \in R^{n+l}$, and by (2.19) we define all elements of the vector $\lambda=\left(\lambda_{1}, \lambda_{2}, \ldots, \lambda_{m+1}, \lambda_{m+2}\right) \in R^{n(m+1)+l}$ through $c$. Substituting the corresponding expressions of $\lambda_{r}, r=\overline{1, m+2}$, into the right-hand side of $(2.6),(2.7)$, and $(2.8)$ we obtain the function $\widetilde{x}(c, t)=x\left(\delta_{m}(\theta), \lambda(c), t\right)$ defined on $[0, T]$ and $\widetilde{\mu}(c)=\mu\left(\delta_{m}(\theta), \lambda(c)\right)$. It follows from Theorems 1,2 , and 3 that the pair $(\widetilde{x}(c, t), \widetilde{\mu}(c))$ is a classical general solution to Equation (2.1). Theorem 4 is proved.

Formulas (2.9)-(2.12), which determine the coefficients and the right-hand sides of the $\delta_{m}(\theta)$ general solution, contain the fundamental matrices $X_{r}(t), r=\overline{1, m}$. As a rule, the construction of fundamental matrices for systems of ordinary differential equations with variable coefficients fails. Therefore, below we propose numerical and approximate methods for constructing a $\delta_{m}(\theta)$ general solution. To do this, we consider the Cauchy problems for ordinary differential equations on sub-intervals:

$$
\frac{d v}{d t}=A(t) v+P(t), \quad v\left(\theta_{r-1}\right)=0, \quad t \in\left[\theta_{r-1}, \theta_{r}\right], \quad r=\overline{1, m},
$$

where $P(t)$ is a square matrix or a vector of dimension $n$ continuous on $[0, T]$.

Denote by $a_{r}(P, t)$ the unique solution to the Cauchy problem (2.20). It is clear that

$$
a_{r}(P, t)=X_{r}(t) \int_{\theta_{r-1}}^{t} X_{r}^{-1}(\tau) P(\tau) d \tau, \quad t \in\left[\theta_{r-1}, \theta_{r}\right], \quad r=\overline{1, m}
$$


Now, taking into account (2.21), we can determine the coefficients and the right-hand sides of the $\delta_{m}(\theta)$ general solution from the equations:

$$
\begin{aligned}
& \alpha_{r r}\left(\delta_{m}(\theta), t\right)=I+a_{r}\left(A+K_{r}, t\right), \quad t \in\left[\theta_{r-1}, \theta_{r}\right], \quad r=\overline{1, m}, \\
& \alpha_{r j}\left(\delta_{m}(\theta), t\right)=a_{r}\left(K_{j}, t\right), \quad t \in\left[\theta_{r-1}, \theta_{r}\right], r \neq j, \quad j=\overline{1, m+1} \text {, } \\
& \alpha_{r}^{0}\left(\delta_{m}(\theta), t\right)=a_{r}\left(A_{0}, t\right), \quad t \in\left[\theta_{r-1}, \theta_{r}\right), \quad r=\overline{1, m}, \\
& \beta_{r}\left(\delta_{m}(\theta), t\right)=a_{r}(f, t), \quad t \in\left[\theta_{r-1}, \theta_{r}\right], \quad r=\overline{1, m} \text {. }
\end{aligned}
$$

In the following numerical method for constructing the $\delta_{m}(\theta)$ general solution, we use the fourth-order Runge-Kutta method to solve the Cauchy problems for ordinary differential equations on sub-intervals. Divide each interval $\left[\theta_{r-1}, \theta_{r}\right]$ into $N_{r}$ parts with step $h_{r}=\left(\theta_{r}-\theta_{r-1}\right) / N_{r}, r=\overline{1, m}$. Suppose that the variable $\hat{t}$ takes only discrete values: $\hat{t}=\theta_{r-1}, \hat{t}=\theta_{r-1}+h_{r}, \ldots, \hat{t}=\theta_{r-1}+$ $\left(N_{r}-1\right) h_{r}, \hat{t}=\theta_{r}, r=\overline{1, m}$, and denote by $\left\{\theta_{r-1}, \theta_{r}\right\}$ the set of such values of $\hat{t}$.

Step 1. Solve the Cauchy problems

$$
\frac{d v}{d t}=A(t) v+\left[A(t)+K_{r}(t)\right], \quad v\left(\theta_{r-1}\right)=0, \quad t \in\left[\theta_{r-1}, \theta_{r}\right],
$$

and find $a_{r}^{h_{r}}\left(A+K_{r}, \hat{t}\right), \hat{t} \in\left\{\theta_{r-1}, \theta_{r}\right\}, r=\overline{1, m}$.

Step 2. Solve the Cauchy problems

$$
\frac{d v}{d t}=A(t) v+K_{j}(t), \quad v\left(\theta_{r-1}\right)=0, t \in\left[\theta_{r-1}, \theta_{r}\right], j \neq r, r=\overline{1, m}, j=\overline{1, m+1},
$$

and determine $a_{r}^{h_{r}}\left(K_{j}, \hat{t}\right), \hat{t} \in\left\{\theta_{r-1}, \theta_{r}\right\}$.

Step 3. Solve the Cauchy problems

$$
\frac{d v}{d t}=A(t) v+A_{0}(t), \quad v\left(\theta_{r-1}\right)=0, \quad t \in\left[\theta_{r-1}, \theta_{r}\right]
$$

and find $a_{r}^{h_{r}}\left(A_{0}, \hat{t}\right), \hat{t} \in\left\{\theta_{r-1}, \theta_{r}\right\}, \quad r=\overline{1, m}$.

Step 4. Solve the Cauchy problems

$$
\frac{d v}{d t}=A(t) v+f(t), \quad v\left(\theta_{r-1}\right)=0, \quad t \in\left[\theta_{r-1}, \theta_{r}\right]
$$

and find $a_{r}^{h_{r}}(f, \hat{t}), \hat{t} \in\left\{\theta_{r-1}, \theta_{r}\right\}, \quad r=\overline{1, m}$.

Then in accordance with $(2.22)-(2.25)$, the numerical $\delta_{m}(\theta)$ general solution of Equation (2.1) is defined as follows:

$$
\begin{aligned}
& x^{h_{r}}\left(\delta_{m}(\theta), \hat{t}, \lambda\right)=\left[I+a_{r}^{h_{r}}\left(A+K_{r}, \hat{t}\right)\right] \lambda_{r}+\sum_{j=1, j \neq r}^{m+1} a_{r}^{h_{r}}\left(K_{j}, \hat{t}\right) \lambda_{j} \\
& \quad+a_{r}^{h_{r}}\left(A_{0}, \hat{t}\right) \lambda_{m+2}+a_{r}^{h_{r}}(f, \hat{t}), \quad \hat{t} \in\left\{\theta_{r-1}, \theta_{r}\right\} \backslash\left\{\theta_{r}\right\}, r=\overline{1, m}, \\
& x^{h_{m}}\left(\delta_{m}(\theta), T, \lambda\right)=\lambda_{m+1}, \quad \mu\left(\delta_{m}(\theta), \lambda\right)=\lambda_{m+2} .
\end{aligned}
$$

Using the Lagrange polynomial [9] with functions $\omega_{r, i}(t)=\omega_{r, i}^{1}(t) / \omega_{r, i}^{2}$

$$
\omega_{r, i}^{1}(t)=\left(t-t_{r-1,0}\right)\left(t-t_{r-1,1}\right) \ldots\left(t-t_{r-1, i-1}\right)\left(t-t_{r-1, i+1}\right) \ldots\left(t-t_{r-1, N_{r}}\right),
$$




$$
\begin{aligned}
\omega_{r, i}^{2}= & \left(t_{r-1, i}-t_{r-1,0}\right)\left(t_{r-1, i}-t_{r-1,1}\right) \ldots\left(t_{r-1, i}-t_{r-1, i-1}\right)\left(t_{r-1, i}-t_{r-1, i+1}\right) \\
& \times \ldots\left(t_{r-1, i}-t_{r-1, N_{r}}\right),
\end{aligned}
$$

where $t_{r, i}=\theta_{r-1}+i h_{r}, r=\overline{1, m}, i=0,1, \ldots, N_{r}$, we get an approximate $\delta_{m}(\theta)$ general solution of Equation (2.1). We determine the approximate coefficients and right-hand sides by the equalities:

$$
\begin{aligned}
& \widetilde{a}_{r}^{h_{r}}\left(A+K_{r}, t\right)=\sum_{i=0}^{N_{r}} a_{r}^{h_{r}}\left(A+K_{r}, t_{r-1, i}\right) \omega_{r, i}(t), \\
& \widetilde{a}_{r}^{h_{r}}\left(K_{j}, t\right)=\sum_{i=0}^{N_{r}} a_{r}^{h_{r}}\left(K_{j}, t_{r-1, i}\right) \omega_{r, i}(t), \quad j \neq r, \\
& \widetilde{a}_{r}^{h_{r}}\left(A_{0}, t\right)=\sum_{i=0}^{N_{r}} a_{r}^{h_{r}}\left(A_{0}, t_{r-1, i}\right) \omega_{r, i}(t), \quad \widetilde{a}_{r}^{h_{r}}(f, t)=\sum_{i=0}^{N_{r}} a_{r}^{h_{r}}\left(f, t_{r-1, i}\right) \omega_{r, i}(t), \\
& r=\overline{1, m}, \quad j=\overline{1, m+1}, \quad t \in\left[\theta_{r-1}, \theta_{r}\right] .
\end{aligned}
$$

Then, the approximate $\delta_{m}(\theta)$ general solution of Equation (2.1) has the form:

$$
\begin{aligned}
& \widetilde{x}^{h}\left(\delta_{m}(\theta), t, \lambda\right)=\left[I+\widetilde{a}_{r}^{h_{r}}\left(A+K_{r}, t\right)\right] \lambda_{r}+\sum_{j=1, j \neq r}^{m+1} \widetilde{a}_{r}^{h_{r}}\left(K_{j}, t\right) \lambda_{j} \\
& +\widetilde{a}_{r}^{h_{r}}\left(A_{0}, t\right) \lambda_{m+2}+\widetilde{a}_{r}^{h_{r}}(f, t), \quad t \in\left[\theta_{r-1}, \theta_{r}\right), \quad r=\overline{1, m}, \\
& \widetilde{x}_{h}\left(\delta_{m}(\theta), T, \lambda\right)=\lambda_{m+1}, \quad \mu\left(\delta_{m}(\theta), \lambda\right)=\lambda_{m+2} .
\end{aligned}
$$

Similarly, solving the Cauchy problems for ordinary differential equations $(2.27)-(2.29)$ by an approximate method, we obtain an approximate $\delta_{m}(\theta)$ general solution of Equation (2.1). The accuracy of the coefficients and the righthand sides of the numerical and approximate $\delta_{m}(\theta)$ general solutions depends on the accuracy of the method used to solve Cauchy problems for ordinary differential equations (2.20).

\section{Algorithms for solving a problem with a parameter for a loaded differential equation}

The $\delta_{m}(\theta)$ general solution allows us to reduce the solvability of problem (2.1), (1.2) to the solvability of a system of linear algebraic equations with respect to $\lambda_{r} \in R^{n}, r=\overline{1, m+2}$. Substituting the appropriate expressions of the $\delta_{m}(\theta)$ general solution into the boundary condition (1.2) and the continuity conditions (2.13) and (2.14), we obtain the system of linear algebraic equations:

$$
\begin{aligned}
& B_{0} \lambda_{m+2}+B \lambda_{1}+C \lambda_{m+1}=d, \quad d \in R^{n+l} \\
& \sum_{j=1, j \neq r+1}^{m+1} \alpha_{r j}\left(\delta_{m}(\theta), \theta_{r}\right) \lambda_{j}-\left[I-\alpha_{r r+1}\left(\delta_{m}(\theta), \theta_{r}\right)\right] \lambda_{r+1}+\alpha_{r}^{0}\left(\delta_{m}(\theta), \theta_{r}\right) \lambda_{m+2} \\
& \quad=-\beta_{r}\left(\delta_{m}(\theta), \theta_{r}\right), \quad r=\overline{1, m}
\end{aligned}
$$


Denote by $Q_{*}\left(\delta_{m}(\theta)\right): R^{n(m+1)+l} \rightarrow R^{n(m+1)+l}$ the matrix corresponding to the left-hand side of system (3.1)-(3.2) and rewrite the system as follows:

$$
Q_{*}\left(\delta_{m}(\theta)\right) \lambda=-F_{*}\left(\delta_{m}(\theta)\right), \quad \lambda \in R^{n(m+1)+l},
$$

where $F_{*}\left(\delta_{m}(\theta)\right)=\left(-d, \beta_{1}\left(\delta_{m}(\theta), \theta_{1}\right), \ldots, \beta_{m}\left(\delta_{m}(\theta), \theta_{m}\right)\right) \in R^{n(m+1)+l}$.

Using the properties of the $\delta_{m}(\theta)$ general solution, it is easy to prove that the solvability of the problem with parameter $(2.1),(1.2)$ is equivalent to the solvability of system (3.3).

Definition 2. A problem with parameter (2.1), (1.2) is called well-posed if for any pair $(f(t), d)$ with $f(t) \in C\left([0, T], R^{n}\right)$ and $d \in R^{n+l}$, it has a unique solution $\left(x^{*}(t), \mu^{*}\right)$, and the inequality

$$
\max \left(\left\|x^{*}\right\|_{1},\left\|\mu^{*}\right\|\right) \leq \chi \max \left(\|f\|_{1},\|d\|\right)
$$

holds, where $\chi$ is a constant independent of $f(t)$ and $d$.

The number $\chi$ is called the constant of the well-posedness of the problem with parameter (2.1), (1.2).

From the well-known statements of linear algebra the following two statements follow.

Theorem 5. The problem with parameter (2.1), (1.2) is solvable if and only if the vector $F_{*}\left(\delta_{m}(\theta)\right)$ is orthogonal to the kernel of the transposed matrix $\left(Q_{*}\left(\delta_{m}(\theta)\right)\right)^{\prime}$, i.e. iff the equality $\left(F_{*}\left(\delta_{m}(\theta)\right), \zeta\right)=0$ holds for $\forall \zeta \in \operatorname{Ker}\left(Q_{*}\left(\delta_{m}(\theta)\right)\right)^{\prime}$, where $(\cdot, \cdot)$ is the inner product in $R^{n(m+1)+l}$.

Theorem 6. The problem with parameter (2.1), (1.2) is well-posed if and only if the matrix $Q_{*}\left(\delta_{m}(\theta)\right)$ is invertible.

To solve the problem with parameter $(2.1),(1.2)$, we propose

\section{Algorithm A:}

I. Construct a $\delta_{m}(\theta)$ general solution $\left(x\left(\delta_{m}(\theta), t, \lambda\right), \mu\left(\delta_{m}(\theta), \lambda\right)\right)$ using the formulas (2.9)-(2.12);

II. Form Equation (3.3) by substituting the corresponding expressions of the $\delta_{m}(\theta)$ general solution into the boundary and continuity conditions;

III. Solve Equation (3.3) and find $\lambda_{r}^{*}, r=\overline{1, m+2}$;

IV. Substitute $\lambda_{r}^{*}, r=\overline{1, m+2}$, into the general solution and obtain a solution to problem (2.1), (1.2).

Theorems 5 and 6 allow us to establish the solvability of the boundary value problem (2.1), (1.2). If the problem is solvable, then using Algorithm $A$ we will find its solution. Moreover, the $\delta_{m}(\theta)$ general solution and estimate $\left\|\left[Q_{*}\left(\delta_{m}(\theta)\right)\right]^{-1}\right\|$ allow us to determine the constant of the well-posedness of problem (2.1), (1.2). Suppose that the matrix $Q_{*}\left(\delta_{m}(\theta)\right)$ is invertible, and there exists a constant $\kappa\left(\delta_{m}(\theta)\right)$ such that

$$
\left\|\left[Q_{*}\left(\delta_{m}(\theta)\right)\right]^{-1}\right\| \leq \kappa\left(\delta_{m}(\theta)\right) .
$$

In this case, Equation (3.3) has a unique solution

$$
\lambda^{*}=-\left[Q_{*}\left(\delta_{m}(\theta)\right)\right]^{-1} F_{*}\left(\delta_{m}(\theta)\right),
$$


and the pair $\left(x^{*}(t), \mu^{*}\right)$, where the function $x^{*}(t)=x\left(\delta_{m}(\theta), \lambda^{*}, t\right)$ and the parameter $\mu^{*}=\mu\left(\delta_{m}(\theta), \lambda^{*}\right)$, is the unique solution to the problem with parameter (2.1), (1.2). Equalities (2.9)-(2.12) and the relations

$$
\begin{aligned}
X_{r}(t) & \int_{\theta_{r-1}}^{t} X_{r}^{-1}(\tau) P(\tau) d \tau=\int_{\theta_{r-1}}^{t} P\left(\tau_{1}\right) d \tau_{1}+\int_{\theta_{r-1}}^{t} A\left(\tau_{1}\right) \int_{\theta_{r-1}}^{\tau_{1}} P\left(\tau_{2}\right) d \tau_{2} d \tau_{1} \\
& +\int_{\theta_{r-1}}^{t} A\left(\tau_{1}\right) \int_{\theta_{r-1}}^{\tau_{1}} A\left(\tau_{2}\right) \int_{\theta_{r-1}}^{\tau_{2}} P\left(\tau_{3}\right) d \tau_{3} d \tau_{2} d \tau_{1}+\ldots
\end{aligned}
$$

(see (14) in [11], p.1152) lead to the estimates:

$$
\begin{aligned}
& \sup _{t \in\left[\theta_{r-1}, \theta_{r}\right)}\left\|\alpha_{r r}\left(\delta_{m}(\theta), t\right)\right\| \leq\left[1+\int_{\theta_{r-1}}^{\theta_{r}}\left\|K_{r}(\tau)\right\| d \tau\right] \exp \left\{\int_{\theta_{r-1}}^{\theta_{r}}\|A(\tau)\| d \tau\right\}-1, \\
& \quad r=\overline{1, m}, \\
& \sup _{t \in\left[\theta_{r-1}, \theta_{r}\right)}\left\|\alpha_{r j}\left(\delta_{m}(\theta), t\right)\right\| \leq \int_{\theta_{r-1}}^{\theta_{r}}\left\|K_{j}(\tau)\right\| d \tau \exp \left\{\int_{\theta_{r-1}}^{\theta_{r}}\|A(\tau)\| d \tau\right\}, \\
& \quad j \neq r, j=\overline{1, m+1}, r=\overline{1, m}, \\
& \sup _{t \in\left[\theta_{r-1}, \theta_{r}\right)}\left\|\alpha_{r}^{0}\left(\delta_{m}(\theta), t\right)\right\| \leq \int_{\theta_{r-1}}^{\theta_{r}}\left\|A_{0}(\tau)\right\| d \tau \exp \left\{\int_{\theta_{r-1}}^{\theta_{r}}\|A(\tau)\| d \tau\right\}, r=\overline{1, m} \\
& \sup _{t \in\left[\theta_{r-1}, \theta_{r}\right)}\left\|\beta_{r}\left(\delta_{m}(\theta), t\right)\right\| \leq \int_{\theta_{r-1}}^{\theta_{r}}\|f(\tau)\| d \tau \exp \left\{\int_{\theta_{r-1}}^{\theta_{r}}\|A(\tau)\| d \tau\right\}, r=\overline{1, m} .(3.6)
\end{aligned}
$$

The right-hand side of Equation (3.3) can now be estimated as follows:

$$
\begin{aligned}
& \left\|F_{*}\left(\delta_{m}(\theta)\right)\right\| \leq \max \left(1, b_{0}\right) \max \left(\|f\|_{1},\|d\|\right) \\
& b_{0}=\max _{r=\overline{1, m}}\left(\theta_{r}-\theta_{r-1}\right) \exp \left\{\int_{\theta_{r-1}}^{\theta_{r}}\|A(\tau)\| d \tau\right\} .
\end{aligned}
$$

Inequalities (3.4), (3.5)-(3.6) and (3.7) lead to the estimate:

$$
\begin{aligned}
& \left\|x^{*}\right\|_{1} \leq \max _{r=1, m}\left[\left(1+\sum_{j=1}^{m+1} \int_{\theta_{r-1}}^{\theta_{r}}\left\|K_{j}(\tau)\right\| d \tau+\int_{\theta_{r-1}}^{\theta_{r}}\left\|A_{0}(\tau)\right\| d \tau\right)\right. \\
& \left.\times \exp \left\{\int_{\theta_{r-1}}^{\theta_{r}}\|A(\tau)\| d \tau\right\}-1\right]\left\|\lambda^{*}\right\|+\max _{r=1, m}\left[\int_{\theta_{r-1}}^{\theta_{r}}\|f(\tau)\| d \tau \exp \left\{\int_{\theta_{r-1}}^{\theta_{r}}\|A(\tau)\| d \tau\right\}\right] \\
& \leq \max \left(1, b_{0}\right)\left[\left(c_{0}-1+k_{0} c_{0}+l_{0} c_{0}\right) \kappa\left(\delta_{m}(\theta)\right)+1\right] \max \left(\|f\|_{1},\|d\|\right), \\
& \left\|\mu^{*}\right\| \leq \kappa\left(\delta_{m}(\theta)\right) \max \left(1, b_{0}\right) \max \left(\|f\|_{1},\|d\|\right),
\end{aligned}
$$

where $c_{0}=\max _{r=\overline{1, m}} \exp \left\{\int_{\theta_{r-1}}^{\theta_{r}}\|A(\tau)\| d \tau\right\}, \quad k_{0}=\sum_{j=1}^{m+1} \max _{r=1, m} \int_{\theta_{r-1}}^{\theta_{r}}\left\|K_{j}(\tau)\right\| d \tau$, $l_{0}=\max _{r=1, m} \int_{\theta_{r-1}}^{\theta_{r}}\left\|A_{0}(\tau)\right\| d \tau$. 
From (3.8) and (3.9), we have

$$
\max \left(\left\|x^{*}\right\|_{1},\left\|\mu^{*}\right\|\right) \leq \chi \max \left(\|f\|_{1},\|d\|\right)
$$

where $\chi=\max \left(1, b_{0}\right) \max \left[\left(c_{0}-1+k_{0} c_{0}+l_{0} c_{0}\right) \kappa\left(\delta_{m}(\theta)\right)+1, \kappa\left(\delta_{m}(\theta)\right)\right]$.

Thus, the problem with parameter (2.1), (1.2) is well-posed with constant $\chi$.

As was noted in Section 2, for Equation (2.1) with the variable matrix $A(t)$, in most cases, the construction of the $\delta_{m}(\theta)$ general solution in the explicit form fails. Therefore, we propose another way to solve problem (2.1), (1.2). This method is based on compilation of a system of linear algebraic equations (3.1)(3.2) and does not require the construction of the $\delta_{m}(\theta)$ general solution. Using relations $(2.22)-(2.25)$, we solve problem $(2.1),(1.2)$ using the following

\section{Algorithm B:}

Step 1. Solve the Cauchy problems for ordinary differential equations

$$
\begin{aligned}
& \frac{d v}{d t}=A(t) v+\left[A(t)+K_{r}(t)\right], \quad v\left(\theta_{r-1}\right)=0, \quad t \in\left[\theta_{r-1}, \theta_{r}\right], \quad r=\overline{1, m}, \\
& \frac{d v}{d t}=A(t) v+K_{j}(t), v\left(\theta_{r-1}\right)=0, t \in\left[\theta_{r-1}, \theta_{r}\right], \quad j \neq r, \quad r=\overline{1, m}, j=\overline{1, m+1}, \\
& \frac{d v}{d t}=A(t) v+A_{0}(t), \quad v\left(\theta_{r-1}\right)=0, \quad t \in\left[\theta_{r-1}, \theta_{r}\right], \quad r=\overline{1, m}, \\
& \frac{d v}{d t}=A(t) v+f(t), \quad v\left(\theta_{r-1}\right)=0, \quad t \in\left[\theta_{r-1}, \theta_{r}\right], \quad r=\overline{1, m},
\end{aligned}
$$

and find $a_{r}\left(A+K_{r}, \theta_{r}\right), a_{r}\left(K_{j}, \theta_{r}\right), a_{r}\left(A_{0}, \theta_{r}\right)$ and $a_{r}\left(f, \theta_{r}\right)$.

Step 2. Form a system of linear algebraic equations

$$
\begin{aligned}
& B_{0} \lambda_{m+2}+B \lambda_{1}+C \lambda_{m+1}=d, \\
& {\left[I+a_{p}\left(A+K_{p}, \theta_{p}\right)\right] \lambda_{p}-\left[I-a_{p}\left(K_{p+1}, \theta_{p}\right)\right] \lambda_{p+1}} \\
& \quad+\sum_{\substack{j=1 \\
j \neq p, j \neq p+1}}^{m+1} a_{p}\left(K_{j}, \theta_{p}\right) \lambda_{j}+a_{p}\left(A_{0}, \theta_{p}\right) \lambda_{m+2}=-a_{p}\left(f, \theta_{p}\right), \quad p=\overline{1, m} .
\end{aligned}
$$

Step 3. Solve system (3.10)-(3.11) and find $\lambda^{*}=\left(\lambda_{1}^{*}, \lambda_{2}^{*}, \ldots, \lambda_{m+1}^{*}, \lambda_{m+2}^{*}\right) \in$ $R^{n(m+1)+l}$. Note that the elements of $\lambda^{*}$ are the values of the function $x^{*}(t)$ at the loaded points of $[0, T]: \lambda_{r}^{*}=x^{*}\left(\theta_{r-1}\right), r=\overline{1, m+1}$, and $\lambda_{m+2}^{*}=\mu^{*}$. Here the pair $\left(x^{*}(t), \mu^{*}\right)$ is a solution to problem $(2.1),(1.2)$.

Step 4. Compose the function $\mathcal{F}_{*}(t)=\sum_{j=1}^{m+1} K_{j}(t) \lambda_{j}^{*}+A_{0}(t) \lambda_{m+2}^{*}+f(t)$. Solve Cauchy problems for ordinary differential equations

$$
\frac{d x}{d t}=A(t) x+\mathcal{F}_{*}(t), \quad x\left[\theta_{r-1}\right]=\lambda_{r}^{*}, \quad t \in\left[\theta_{r-1}, \theta_{r}\right]
$$

and determine the values of the solution $x^{*}(t)$ at the remaining points of $\left[\theta_{r-1}, \theta_{r}\right], r=\overline{1, m}$. 
In Algorithm B, Cauchy problems for ordinary differential equations on subintervals are the main auxiliary problems. Using approximate methods for solving these problems leads to approximate methods for solving problem (2.1), (1.2). Numerical methods used for solving Cauchy problems for ordinary differential equations lead to numerical methods for solving the problem (2.1), $(1.2)$.

\section{Interrelation between problems with parameter (1.1)- (1.2) and (2.1), (1.2)}

Let us return to the consideration of the problem with parameter (1.1)-(1.2).

Definition 3. A problem with parameter (1.1)-(1.2) is called well-posed if for any pair $(f(t), d)$ with $f(t) \in C\left([0, T], R^{n}\right)$ and $d \in R^{n+l}$, it has a unique solution $\left(x^{*}(t), \mu^{*}\right)$, and the inequality $\max \left(\left\|x^{*}\right\|_{1},\left\|\mu^{*}\right\|\right) \leq \gamma \cdot \max \left(\|f\|_{1},\|d\|\right)$, where $\gamma$ is a constant, independent of $f(t)$ and $d$, holds.

The number $\gamma$ is called the constant of the well-posedness of the problem with parameter (1.1)-(1.2).

Consider the following problem with parameter for the loaded differential equation:

$$
\begin{aligned}
\frac{d y}{d t}= & A(t) y+\sum_{j=1}^{N} \int_{2(j-1) h}^{2 j h} K(t, \tau) d \tau\left\{\frac{1}{6} y[2(j-1) h]+\frac{4}{6} y[(2 j-1) h]+\frac{1}{6} y[2 j h]\right\} \\
& +A_{0}(t) \nu+f(t), \quad t \in(0, T), \quad y \in R^{n} \\
B_{0} \nu & +B y(0)+C y(T)=d, \quad d \in R^{n+l} .
\end{aligned}
$$

Set $\alpha=\max _{t \in[0, T]}\|A(t)\|, \beta=\max _{(t, \tau) \in[0, T] \times[0, T]}\|K(t, \tau)\|$, and $\alpha_{0}=\max _{t \in[0, T]}\left\|A_{0}(t)\right\|$.

The following two statements provide a connection between the original problem with the parameter for the integro-differential equation (1.1)-(1.2) and the approximating problem with the parameter for the loaded differential equation (4.1)-(4.2).

Theorem 7. Suppose that the problem with parameter (1.1)-(1.2) is well-posed with the constant $\gamma$, and let the inequality

$$
q_{1}(h)=\frac{2}{3} \beta T h\left[\left(\alpha+\beta T+\alpha_{0}\right) \gamma+1\right]<1
$$

be true. Then the problem with parameter (4.1)-(4.2) is well-posed with the constant $\chi=3 \gamma /\left(3-2 \beta T h\left[\left(\alpha+\beta T+\alpha_{0}\right) \gamma+1\right]\right)$, and

$$
\max \left(\left\|y^{*}-x^{*}\right\|_{1},\left\|\nu^{*}-\mu^{*}\right\|\right) \leq \frac{2 \beta T \gamma\left[\left(\alpha+\beta T+\alpha_{0}\right) \gamma+1\right] h}{3-2 \beta T h\left[\left(\alpha+\beta T+\alpha_{0}\right) \gamma+1\right]} \max \left(\|f\|_{1},\|d\|\right)
$$

where $\left(y^{*}(t), \nu^{*}\right)$ and $\left(x^{*}(t), \mu^{*}\right)$ are solutions to problems with parameter $(4.1)$ (4.2) and (1.1)-(1.2), respectively. 
Theorem 8. Let the problem with parameter (2.1), (1.2) be well-posed with constant $\chi$, and the inequality $q_{2}(h)=\frac{2}{3} \beta T h\left[\left(\alpha+\beta T+\alpha_{0}\right) \chi+1\right]<1$ be true. Then the control problem (1.1)-(1.2) is well-posed with the constant $\gamma=3 \chi /\left(3-2 \beta T h\left[\left(\alpha+\beta T+\alpha_{0}\right) \chi+1\right]\right)$, and

$$
\max \left(\left\|x^{*}-y^{*}\right\|_{1},\left\|\mu^{*}-\nu^{*}\right\|\right) \leq \frac{2 \beta T \chi\left[\left(\alpha+\beta T+\alpha_{0}\right) \chi+1\right] h}{3-2 \beta T h\left[\left(\alpha+\beta T+\alpha_{0}\right) \chi+1\right]} \max \left(\|f\|_{1},\|d\|\right),
$$

where $\left(x^{*}(t), \mu^{*}\right)$ and $\left(y^{*}(t), \nu^{*}\right)$ are solutions to problems with parameter $(1.1)$ (1.2) and (4.1)-(4.2), respectively.

Now let us approximate the problem with parameter (1.1)-(1.2) by the problem with parameter:

$$
\begin{aligned}
\frac{d z}{d t}= & A(t) z+\frac{h}{3} \sum_{j=1}^{N}\{K(t, 2(j-1) h) z[2(j-1) h]+4 K(t,(2 j-1) h) z[(2 j-1) h] \\
& +K(t, 2 j h) z[2 j h]\}+A_{0}(t) v+f(t), \quad t \in(0, T), \quad z \in R^{n} \\
B_{0} v & +B z(0)+C z(T)=d, \quad d \in R^{n}
\end{aligned}
$$

Assume that $\omega(K, 2 h, t)=\max _{\substack{\tau^{\prime}, \tau^{\prime \prime} \in[0, T] \\\left|\tau^{\prime}-\tau^{\prime \prime}\right|<2 h}}\left\|K\left(t, \tau^{\prime}\right)-K\left(t, \tau^{\prime \prime}\right)\right\|$ and $\omega_{0}(K, 2 h)=$ $\max _{t \in[0, T]} \omega(K, 2 h, t)$.

Theorem 9. Suppose that a problem with parameter (1.1)-(1.2) is well-posed with a constant $\gamma$. Then there exists a number $h_{0}>0$ such that for all $h \in$ $\left(0, h_{0}\right]: 2 N h=T$ problem $(4.3)-(4.4)$ is well-posed with constant $\chi_{0}=4 \gamma$, and

$$
\begin{aligned}
& \max \left(\left\|z^{*}-x^{*}\right\|_{1},\left\|v^{*}-\mu^{*}\right\|\right) \\
& \quad \leq \gamma T\left\{\frac{4}{3} \beta\left[\left(\alpha+\beta T+\alpha_{0}\right) \gamma+1\right] h+8 \gamma \omega_{0}(K, 2 h)\right\} \max \left(\|f\|_{1},\|d\|\right),
\end{aligned}
$$

where $\left(z^{*}(t), v^{*}\right)$ and $\left(x^{*}(t), \mu^{*}\right)$ are solutions to problems with parameter $(4.3)$ (4.4) and (1.1)-(1.2), respectively.

Theorem 10. Suppose that there are numbers $h_{0}>0$ and $\chi_{0}>0$ such that the problem with parameter (4.3)-(4.4) is well-posed with the constant $\chi_{0}$ for all $h \in\left(0, h_{0}\right]: 2 N h=T$. Then the problem with parameter $(1.1)-(1.2)$ is well-posed with the constant $\gamma=4 \chi_{0}$, and

$$
\begin{aligned}
& \max \left(\left\|x^{*}-z^{*}\right\|_{1},\left\|\mu^{*}-v^{*}\right\|\right) \\
& \leq 2 \chi_{0} T\left\{\frac{4}{3} \beta\left[2 \chi_{0}\left(\alpha+\beta T+\alpha_{0}\right)+1\right] h+\chi_{0} \omega_{0}(K, 2 h)\right\} \max \left(\|f\|_{1},\|d\|\right),
\end{aligned}
$$

where $\left(x^{*}(t), \mu^{*}\right)$ and $\left(z^{*}(t), v^{*}\right)$ are solutions to problems with parameter $(1.1)$ (1.2) and (4.3)-(4.4), respectively.

Taking into account the properties of problem (1.1)-(1.2), the proofs of Theorems $7-10$ are similar to the proofs of Theorems $4.1-4.4$ in [15]. 


\section{A numerical method for solving the problem with parameter (1.1)-(1.2)}

The methods proposed in this Section are based on approximating the problem with parameter (1.1)-(1.2) by problems with the parameter for loaded differential equations and on the Algorithm $B$ presented in Section 4.

Take $h>0: 2 N h=T$ and introduce the notation: $\widetilde{K}_{j}(t)=\frac{4 h}{3} K(t,(j-1) h)$ for even $j$ and $\quad \widetilde{K}_{j}(t)=\frac{2 h}{3} K(t,(j-1) h)$ for odd $j$, where $j=2,3, \ldots, 2 N$, and $\widetilde{K}_{1}(t)=\frac{h}{3} K(t, 0), \quad \widetilde{K}_{2 N+1}(t)=\frac{h}{3} K(t, T)$. Then the approximating problem with parameter $(4.3),(4.4)$ can be written as follows:

$$
\begin{aligned}
& \frac{d z}{d t}=A(t) z+\sum_{j=1}^{2 N+1} \widetilde{K}_{j}(t) z[(j-1) h]+A_{0}(t) v+f(t), t \in(0, T), z \in R^{n}, v \in R^{l} \\
& B_{0} v+B z(0)+C z(T)=d, \quad d \in R^{n+l}
\end{aligned}
$$

The loaded differential equation (5.1) is the Equation (2.1) with $m=2 N$, $\theta_{j-1}=(j-1) h, j=\overline{1,2 N+1}$. Denote by $\delta_{2 N}(h)$ the new general solution to Equation (5.1).

The results of Section 2 establish the relationship between the well-posednesses of problems with parameter (1.1)-(1.2) and (5.1)-(5.2). Since the uniform continuity of $K(t, \tau)$ on $[0, T] \times[0, T]$ leads to $\lim _{h \rightarrow 0} \omega_{0}(K, 2 h)=0$, estimates (4.5) and (4.6) yield uniform convergence of the solution of the approximating problem with parameter $(5.1)-(5.2)$ to the solution of the original problem with parameter (1.1)-(1.2) on $[0, T]$ as $h \rightarrow 0$. In theorems of Section 4, we only need the continuity of $A(t), A_{0}(t)$, and $f(t)$ on $[0, T]$ and $K(t, \tau)$ on $[0, T] \times[0, T]$, respectively. In the following statement, with greater smoothness of these data, the accuracy of approximating the solution of the problem with parameter (1.1)-(1.2) by the solution of the problem with parameter (5.1)-(5.2) is obtained.

Theorem 11. Suppose the following conditions are met: a) problem (1.1)(1.2) is well-posed with constant $\gamma ; b)$ the matrices $A(t), A_{0}(t)$ and the vector $f(t)$ have continuous derivatives $A^{(k)}(t), A_{0}^{(k)}(t)$ and $f^{(k)}(t), k=\overline{1,3}$, on $[0, T]$; $c)$ the matrix $K(t, \tau)$ has continuous partial derivatives $K_{t, \tau}^{(k, i)}(t, \tau), k=\overline{1,3}$, $i=\overline{1,4}$, on $[0, T] \times[0, T]$. Then, there exists $h_{0}>0$ such that for all $h \in$ $\left(0, h_{0}\right]: 2 N h=T$, the problem with parameter $(5.1)-(5.2)$ has a unique solution $\left(z^{*}(t), v^{*}\right)$, and

$$
\max \left(\left\|z^{*}-x^{*}\right\|_{1},\left\|v^{*}-\mu^{*}\right\|\right) \leq \frac{4 \gamma T}{180} \max _{(t, \tau) \in[0, T] \times[0, T]}\left\|\frac{\partial^{4}}{\partial \tau^{4}}\left[K(t, \tau) x^{*}(\tau)\right]\right\| h^{4},
$$

where $\left(x^{*}(t), \mu^{*}\right)$ is the unique solution to the problem with parameter (1.1)$(1.2)$.

We construct the $\delta_{2 N}(h)$ general solution to the loaded differential equation 
with parameter (5.1):

$$
\begin{aligned}
& z\left(\delta_{2 N}(h), t, \lambda\right)=\left[I+a_{r}\left(A+\widetilde{K}_{r}, t\right)\right] \lambda_{r}+\sum_{j=1, j \neq r}^{2 N+1} a_{r}\left(\widetilde{K}_{j}, t\right) \lambda_{j}+a_{r}(f, t), \\
& \quad t \in[(r-1) h, r h), r=\overline{1,2 N}, \\
& z\left(\delta_{2 N}(h), T, \lambda\right)=\lambda_{2 N+1}, \quad v\left(\delta_{2 N}(h), \lambda\right)=\lambda_{2 N+2} .
\end{aligned}
$$

Substituting the corresponding expressions of $z\left(\delta_{2 N}(h), t, \lambda\right)$ and $v\left(\delta_{2 N}(h), \lambda\right)$ into the boundary condition (5.2) and the continuity conditions for solution at the points $t_{s}=s h, s=1,2, \ldots, 2 N$, then multiplying the boundary condition by $h>0$, we obtain the system of linear algebraic equations:

$$
\begin{aligned}
& h B_{0} \lambda_{2 N+2}+h B \lambda_{1}+h C \lambda_{2 N+1}=h d, \\
& {\left[I+a_{p}\left(A+\widetilde{K}_{p}, p h\right)\right] \lambda_{p}-\left[I-a_{p}\left(\widetilde{K}_{p+1}, p h\right)\right] \lambda_{p+1}+\sum_{\substack{j=1 \\
j \neq p, j \neq p+1}}^{2 N+1} a_{p}\left(\widetilde{K}_{j}, p h\right) \lambda_{j}} \\
& \quad+a_{p}\left(A_{0}, p h\right) \lambda_{2 N+2}=-a_{p}(f, p h), \quad p=\overline{1,2 N}^{\text {. }}
\end{aligned}
$$

Denote by $Q_{*}\left(\delta_{2 N}(h)\right): R^{n(2 N+1)+l} \rightarrow R^{n(2 N+1)+l}$ the matrix corresponding to the left-hand side of system (5.4)-(5.5) and rewrite down the system as follows:

$$
Q_{*}\left(\delta_{2 N}(h)\right) \lambda=-F_{*}\left(\delta_{2 N}(h)\right), \quad \lambda \in R^{n(2 N+1)+l},
$$

where $F_{*}\left(\delta_{2 N}(h)\right)=\left(-h d, a_{1}(f, h), a_{2}(f, 2 h), \ldots, a_{2 N}(f, 2 N h)\right) \in R^{n(2 N+1)+l}$.

Let us consider issues related to the qualitative properties of Equation (5.6). As indicated in $[7,9,10]$, the conditionality number is one of the important characteristics for a system of linear algebraic equations. This number, defined as the product of the norms of system's matrix and its inverse, shows how the variations of coefficients and the right sides of the system affect the variation of system's solution. To determine the conditionality number for Equation (5.6), we must estimate $\left\|Q_{*}\left(\delta_{2 N}(h)\right)\right\|$ and $\left\|\left[Q_{*}\left(\delta_{2 N}(h)\right)\right]^{-1}\right\|$. Using inequalities (3.7)-(3.9) and taking into account that $\max _{t \in[0, T]}\|A(t)\|=\alpha$, $\max _{t \in[0, T]}\left\|A_{0}(t)\right\|=\alpha_{0}, \max _{(t, \tau) \in[0, T] \times[0, T]}\|K(t, \tau)\|=\beta$, we have:

$$
\begin{aligned}
& \left\|Q_{*}\left(\delta_{2 N}(h)\right)\right\| \leq \max \left[h\left(\left\|B_{0}\right\|+\|B\|+\|C\|\right), 2\right. \\
& \left.\quad+\max _{p=1,2 N}\left(\left\|a_{p}\left(A+\widetilde{K}_{p}, p h\right)\right\|+\sum_{j=1, j \neq p}^{2 N+1}\left\|a_{p}\left(\widetilde{K}_{j}, p h\right)\right\|+\left\|a_{p}\left(A_{0}, p h\right)\right\|\right)\right] \\
& \leq \max \left[h\left(\left\|B_{0}\right\|+\|B\|+\|C\|\right), 1+\left(1+\beta T h+\alpha_{0} h\right) \exp (\alpha h)\right] .
\end{aligned}
$$

Hence, the norm of $Q_{*}\left(\delta_{2 N}(h)\right)$ does not depend on $2 N$, the number of partition.

Theorem 12. Let the problem (5.1)-(5.2) be well-posed with the constant $\chi_{0}$. Then there exists a number $h_{1}>0$ such that for all $h \in\left(0, h_{1}\right]: 2 N h=T$, 
the $(n(2 N+1)+l) \times(n(2 N+1)+l)$ matrix $Q_{*}\left(\delta_{2 N}(h)\right)$ is invertible, and the estimate

$$
\left\|\left[Q_{*}\left(\delta_{2 N}(h)\right)\right]^{-1}\right\| \leq 2 \chi_{0} / h
$$

is true.

The proofs of Theorems 11 and 12 with slight modifications are similar to the proofs of Theorems 4.1 and 4.2, respectively, in [4].

So, if problem (5.1)-(5.2) is well-posed with the constant $\chi_{0}$, then inequalities (5.7) and (5.8) yield the estimate

$$
\begin{aligned}
& \operatorname{cond}_{\infty} Q_{*}\left(\delta_{2 N}(h)\right)=\left\|Q_{*}\left(\delta_{2 N}(h)\right)\right\| \cdot\left\|\left[Q_{*}\left(\delta_{2 N}(h)\right)\right]^{-1}\right\| \\
& \quad \leq \frac{2 \chi_{0}}{h} \max \left[h\left(\left\|B_{0}\right\|+\|B\|+\|C\|\right), 1+\left(1+\beta T h+\alpha_{0} h\right) \exp (\alpha h)\right]
\end{aligned}
$$

for $h \in\left(0, h_{1}\right]: 2 N h=T$. As can be seen from (5.9), the conditionality number for system (5.6) increases linearly with respect to $2 N$ if the problem with parameter (1.1)-(1.2) is well-posed. This property ensures stability when solving system (5.4)-(5.5) (see [7]).

In [4] we also propose a numerically approximate method for solving Cauchy problems for ordinary differential equations on subintervals, which is illustrated by numerical examples. The authors can present numerical results obtained using MathCad15.

\section{Acknowledgements}

This research was supported and partially supported by Ministry of Education and Science of Republic Kazakhstan Grants No. AP09258829, AP09259618 and AP09259414 respectively. The authors thank the reviewers for a thorough reading of the manuscript and useful suggestions that have allowed us to improve this article.

\section{References}

[1] V.M. Abdullaev and K.R. Aida-zade. Numerical method of solution to loaded nonlocal boundary value problems for ordinary differential equations. Comput. Math. Math. Phys., 54(10):1096-1109, 2014. https://doi.org/10.1134/S0965542514070021.

[2] V.M. Abdullaev and K.R. Aida-zade. On the numerical solution of loaded systems of ordinary differential equations with nonseparated multipoint and integral conditions. Numer. Anal. Appl., 7(1):1-17, 2014. https://doi.org/10.1134/S1995423914010017.

[3] M.U. Akhmetov, A. Zafer and R.D. Sejilova. The control of boundary value problems for quasilinear impulsive integro-differential equations. Nonl. Anal.: Theory, Methods \& Appl., 48(1):271-286, 2002.

[4] A.T. Assanova, E.A. Bakirova and Z.M. Kadirbayeva. Numerical solution to a control problem for the integro-differential equations. Comput. Math. Math. Phys., 60(2):203-221, 2020. https://doi.org/10.1134/S0965542520020049. 
[5] A.T. Assanova, A.E. Imanchiyev and Z.M. Kadirbayeva. Numerical solution of systems of loaded ordinary differential equations with multipoint conditions. Comput. Math. Math. Phys., 58(4):508-516, 2018. https://doi.org/10.1134/S096554251804005X.

[6] A.T. Assanova and Zh.M. Kadirbayeva. On the numerical algorithms of parametrization method for solving a two-point boundary-value problem for impulsive systems of loaded differential equations. Comput. and Appl. Math., 37(4):4966-4976, 2018. https://doi.org/10.1007/s40314-018-0611-9.

[7] K.I. Babenko. Fundamentals of Numerical Analysis. Nauka, Moscow, 1986. (in Russian)

[8] A.A. Boichuk and A.M. Samoilenko. Generalized inverse operators and Fredholm boundary-value problems. SP, Utrecht, Boston, 2004.

[9] H. Brunner. Collocation methods for Volterra integral and related functional equations. Cambridge University Press, Cambridge, 2004.

[10] H. Cohen. Numerical approximation methods. Springer, New York, 2011.

[11] D.S. Dzhumabaev. A method for solving the linear boundary value problem for an integro-differential equation. Comput. Math. Math. Phys., 50(7):1150-1161, 2010. https://doi.org/10.1134/S0965542510070043.

[12] D.S. Dzhumabaev. An algorithm for solving the linear boundary value problem for an integro-differential equation. Comput. Math. Math. Phys., 53(6):736-758, 2013. https://doi.org/10.1134/S0965542513060067.

[13] D.S. Dzhumabaev. Necessary and sufficient conditions for the solvability of linear boundary-value problems for the fredholm integro-differential equation. Ukr. Math. J., 66(8):1200-1219, 2015. https://doi.org/10.1007/s11253-015-1003-6.

[14] D.S. Dzhumabaev. On one approach to solve the linear boundary value problems for Fredholm integro-differential equations. J. Comput. Appl. Math., 294(2):342357, 2016. https://doi.org/10.1016/j.cam.2015.08.023.

[15] D.S. Dzhumabaev. Computational methods of solving the boundary value problems for the loaded differential and fredholm integro-differential equations. Math. Meth. Appl. Sci., 41(4):1439-1462, 2018. https://doi.org/10.1002/mma.4674.

[16] D.S. Dzhumabayev. Criteria for the unique solvability of a linear boundary-value problem for an ordinary differential equation. USSR Comput. Math. Math. Phys., 29(1):34-46, 1989.

[17] R. Kangro and E. Tamme. On fully discrete collocation methods for solving weakly singular integro-differential equations. Math. Model. Anal., 15(1):69-82, 2010. https://doi.org/10.3846/1392-6292.2010.15.69-82.

[18] A.M. Krall. The development of general differential and general differentialboundary systems. Rocky Mountain J. Math., 5(4):493-542, 1975.

[19] A.Yu. Luchka and O.B. Nesterenko. Projection method for the solution of integro-differential equations with restrictions and control. Nonl. Oscil., 11(2):219-228, 2008. https://doi.org/10.1007/s11072-008-0025-5.

[20] A.Yu. Luchka and O.B. Nesterenko. Construction of solution of integrodifferential equations with restrictions and control by projection-iterative method. Nonl. Oscil., 12(1):85-93, 2009. https://doi.org/10.1007/s11072-0090061-9. 
[21] A.M. Nakhushev. Boundary value problems for loaded integral-differential equations of hyperbolic type and their applications to the soil moisture forecast. Differencial'nye Uravneniya, 15(1):96-105, 1979.

[22] A.M. Nakhushev. On an approximate method of solving boundary value problems for differential equations and its applications to the dynamics of soil moisture and groundwater. Differencial'nye Uravneniya, 18(1):72-81, 1982. (in Russian)

[23] A.M. Nakhushev. Loaded equations and their applications. Nauka, Moscow, 2012. (in Russian)

[24] O.B. Nesterenko. Modified projection-iterative method for weakly nonlinear integro differential equations with parameters. J. Math. Sci., 198(3):328-335, 2014. https://doi.org/10.1007/s10958-014-1793-3.

[25] A. Pedas and E. Tamme. Product integration for weakly singular integro-differential equations. Math. Model. Anal., 16(1):153-172, 2011. https://doi.org/10.3846/13926292.2011.564771. 